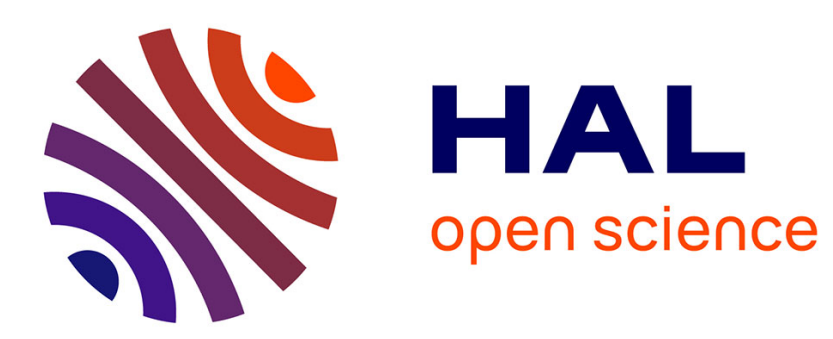

\title{
Packing of ovaloïd atoms in actinide structures
}

\author{
V. Dmitriev, B. Mettout, P. Toledano
}

\section{To cite this version:}

V. Dmitriev, B. Mettout, P. Toledano. Packing of ovaloïd atoms in actinide structures. Journal de Physique IV Proceedings, 1994, 04 (C3), pp.C3-163-C3-167. 10.1051/jp4:1994323 . jpa-00252520

\section{HAL Id: jpa-00252520 https://hal.science/jpa-00252520}

Submitted on 1 Jan 1994

HAL is a multi-disciplinary open access archive for the deposit and dissemination of scientific research documents, whether they are published or not. The documents may come from teaching and research institutions in France or abroad, or from public or private research centers.
L'archive ouverte pluridisciplinaire HAL, est destinée au dépôt et à la diffusion de documents scientifiques de niveau recherche, publiés ou non, émanant des établissements d'enseignement et de recherche français ou étrangers, des laboratoires publics ou privés. 


\title{
Packing of ovaloïd atoms in actinide structures
}

\author{
V. DMITRIEV, B. METTOUT and P. TOLEDANO
}

Laboratoire des Transitions de Phases, 33 rue Saint-Leu, 80000 Amiens, France

\begin{abstract}
Résumé.
The atomistic mechanisms which result in the structures of eleven actinide elements are deduced from a parent bcc structure. The rich polymorphism and idiosyncratic structures found in uranium, neptunium and plutonium are interpreted in terms of close packings of ovaloïd atoms. The incommensurate structure found in $\alpha-U$ is discussed in this framework.
\end{abstract}

In addition to the standard bcc, fcc and hcP structures which are found all over the Periodic Table, actinide crystals display a number of low symmetry structures which are a prominent feature of this family of elements ${ }^{1,2}$. Thus, $\alpha-$ neptunium and the $\beta$-phases of plutonium and uranium constitute idiosyncratic structures, i.e. they are unique among all known structures. Besides, the incommensurate phase observed in $\alpha-U$, is the sole case of structural incommensurability reported in crystals of the elements 3,4 .

The aim of the present work is to propose a unified description of the structures in actinide crystals using a recent theory of reconstructive transitions of the displacive type ${ }^{2}$. More precisely we will show that all the structures, except one, found in crystals of actinide elements can be derived from the same parent bcc structure via a small number of atomistic mechanisms.

The connections of the low-symmetry structures found in actinides with the bcc parent structure is shown in Fig. 1. One can see that three main situations can be distinguished.

1. In the lighter actinides, namely actinium, thorium and protactinium, the more complete sequence of structures $\operatorname{Im} 3 \mathrm{~m}(Z=1) \rightarrow I 4 / \mathrm{mmm}(Z=1) \rightarrow \mathrm{Fm} 3 \mathrm{~m}(Z=1)$, which is fully realized in $\mathrm{Pa}$, and only partially in $\mathrm{Th}$ and $\mathrm{Ac}$ corresponds to the Bain deformation mechanism, as it was reformulated recently at the macroscopic and atomistic levels.

2. A large variety of structures are found in the phase diagrams of the heavier actinide elements (americium, curium, berkelium, californium, einsteinium,....), the more complete set of five distinct structures having been identified in Am', which are denoted AmI (P6 $/ \mathrm{mmc}, Z=4)$, AmII (Fm3m, $Z=1)$, AmIII $(\mathrm{P} 21 / \mathrm{m}, Z=4)$, AmIV/CmCm, $Z=2$ ) and $\mathrm{AmV}$ ( $\mathrm{Im} 3 \mathrm{~m}, \mathrm{Z}=1$ ). The AmV $\rightarrow$ AmII transformation, which takes place with increasing temperature at $\mathbf{T}_{\mathrm{A}}=1352 \mathrm{z}$ and atmospheric pressure corresponds to the direct Bain deformation. The sequence of phases AmI $\rightarrow$ AmIII AmIV, which is found below $800 \mathrm{k}$, with increasing pressure ${ }^{9}$, can be understood in the framework of a variant of the Burgers mechanism proposed in ref 6 and represented in Fig. 2.a.

Except the bcc parent structure, the same phases as in Am are observed in Cf [10]. In $\mathrm{Cm}$ and $\mathrm{Bk}$ three of the Am structures are found ${ }^{11}$ (fcc, double-hcp, and orthorhombic). In Es only the fcc structure of AmII is presently known.

3. Let us now focus on the complex and puzzling structures which are found in 


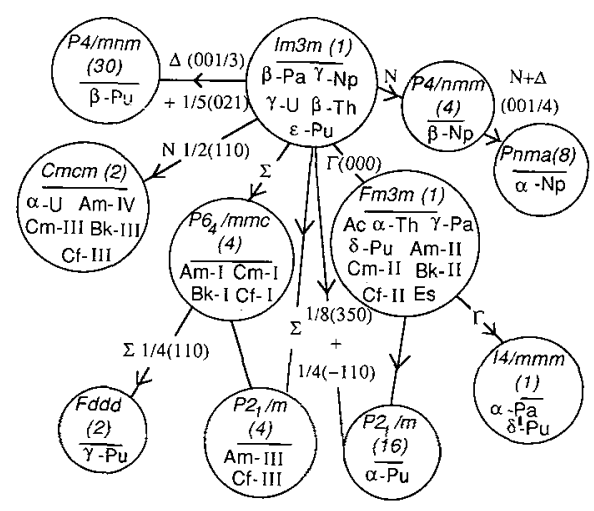

Fig. 1 : Symmetry relationship between the structures in actinide elements. The Brillouin-zone points associated with the transformations are indicated on the lines connecting the phases.

intermediate actinides : uranium, neptunium and plutonium. In their pressuretemperature phase diagrams ${ }^{2}$ the bcc structure, denoted respectively $\gamma-\mathrm{U}, \gamma-\mathrm{N}_{\mathrm{p}}$, and $\varepsilon-P u i s$ stable below the melting line and thus appears as the natural parent structure for the three elements, as is it assumed in our approach for all the actinide group. Two sequences of phases, namely $\varepsilon-\mathrm{Pu}+\delta^{\prime}-\mathrm{Pu}(\mathrm{I} 4 / \mathrm{mmm}, \mathrm{Z}=1) \rightarrow \delta$ $\mathrm{Pu}$ ( fcc, $Z=1$ ) and $\gamma-U(\operatorname{Im} 3 \mathrm{~m}, Z=1) \rightarrow \alpha-U(\mathrm{CmCm}, Z=2)$ can again be interpreted in the framework of the Bain deformation and Burgers mechanisms and will not be discussed further. All the remaining structures found in $U, N_{P}$ and $P u$ require specific mechanisms and will now be described separately.

The crystallographic description of the $\beta$-phase of uranium ${ }^{11}\left(\mathrm{P}_{2}\left(\mathrm{mnm}_{;} \mathrm{Z}=30\right)\right.$ which is found between $941 \mathrm{~K}$ and $1048 \mathrm{~K}$ can be obtained as the result of a displacive mechanism from the $\gamma-U$ phase, as illustrated in Figs. 2b, $c ; d$, e. It can be summarized as follows : six successive layers of the (100) type, of the bcc bi-layer structure $A_{1} B_{1} A_{2} B_{2} A_{3} B_{3}$ generate the four-layer $A B A C B-U$ structure in two correlated steps : i) each of the two couples of bcc layers $\left(B_{3}+A_{2}\right)$ and $\left(A_{3}+B_{3}\right)$ collapse and form respectively the $B$ and $C B-U$ layers: $i i)^{1}$ one atom of each of the $A_{1}$ and $\mathrm{B}_{2}$ bcc layers are ejected from the layers and complete the $B$ and $C$ layers the rêmaining atoms of $A_{1}$ and $B_{2}$ constituting the two A-layers of $B-U$, which are shifted one with respect to the other by $\left(\frac{a}{2} \frac{a}{2} c\right)$. Figs. $2 b$, $c$ and $d$ decompose the corresponding displacements of the uranium atoms from their bce to their $\beta-U$ positions.

The formation of the two low-symmetry phases of neptunium ${ }^{12}$ namely $B-N_{p}(P 4 / n m m, Z$ $=4$ ) and $\alpha-N_{\text {. }}$ (Pnma, $Z=8$ ) which appear consecutively on cooling below bcc $\gamma-N$ can be understood on the basis of standard symmetry considerations, as they are related by a group-subgroup relationship to the bcc structure. The connection between the $\beta$ and $\gamma$ unit-cells is shown in Fig. 3.a. In the same figure are indicated the displacements of the $N_{p}$ atomes which transform successively $\gamma-N_{p}$ in $\beta-N_{p}$ and $\alpha-N$ One can see that the tetragonal structure corresponds to an antiparallel $p_{\text {shifting }} p^{p}$ along the $\mathrm{z}$ axis, of two among the four $\mathrm{N}_{\mathrm{p}}$ atoms contained in each tetragonal unit-cell. The formation of the orthorhombic structure requires an additional antiparallel shifting of all the 8 atoms contained in each orthorhombic cell, simultaneously along the c-tetragonal and b-orthorhombic axes.

At last we will consider the three idiosyncratic structures ${ }^{1,2}$ of plutonium, namely $\gamma-\mathrm{Pu}$ (Fddd, $Z=2), \beta-\mathrm{Pu}(\mathrm{I} 2 / \mathrm{m}, \quad \mathrm{Z}=17)$ and $\alpha-\mathrm{Pu}(\mathrm{P2} / \mathrm{m}, \mathrm{Z}=16)$ which appear in the preceding order on cooling. The four-layered orthorhombic $\gamma-\mathrm{Pu}$ 
(a)

(b)

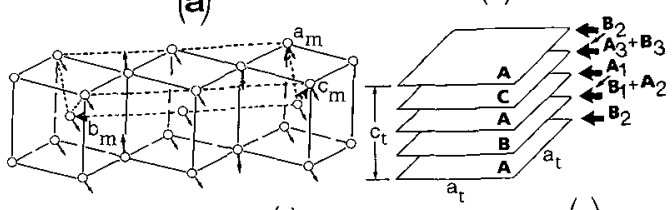

(c) (d)

(e)

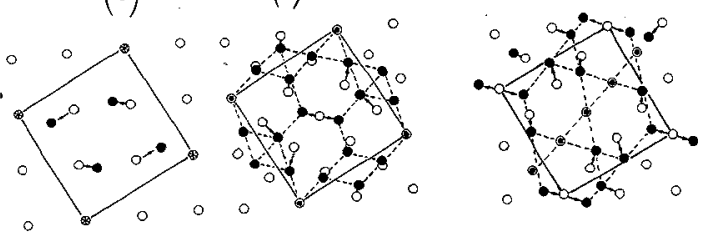

Fig. 2 : Shifting mechanism from the bcc unit.cell of AmV (small cubes in thick lines) to orthorhombic AmIV (short arrows) and monoclinic AmIII (long arrows). The dashed lines represent the basic translations of the monoclinic unit-cell in direction $\vec{b}_{m}$. (b) to (e) Transformation mechanism from $\gamma-U$ to $B-U$ structure. (b) schematic change from the $A_{1} B_{1} A_{2} B_{2} A_{3} B_{3}$ to the $A B A C$ sequence of layers. (c), (d) and (e) represent the in plane shifts for the $A, B$ and $C B-U$ layers. Open and solid circles in (c) indicate the atoms ejected from $A_{1}$. Centered circles in (d) and (e) underline the coincidence of the collapsed layers. The dashed lines illustrate the characteristic in-plane motif.

arises just below bcc $\varepsilon-\mathrm{Pu}$. However, due to its pseudohexagonal character the mechanism of formation can be better viewed by considering an intermediate virtual double hop structure of the AmI type. Thus; the mechanism generating $\gamma-P u$ can be simply described, as shown in Fig.3.b, by an antiparallel $\frac{a}{4}$ shifting of two pairs of alternate planes forming the four layer double hcp structure. One pair of planes is shif ted in the $\{001\}$ bcc direction, whereas the other pair shifts in the perpendicular [110! direction.

As for $\gamma-\mathrm{Pu}$, the displacive mechanism which leads to the formation of $\alpha-\mathrm{Pu}$, can be more easily represented with respect to the intermediate $\delta-\mathrm{Pu}$ structure. The shifts which transform the fcc unit-cell of $\delta-\mathrm{Pu}$ into the sixteen-fold monoclinic $\alpha-\mathrm{Pu}$ unit-cell, are represented in Fig. 3.c. Their non-symmetric directions are consistent with the drastic lowering of point-group symmetry between the two structures.

The group-theoretical and macroscopic features associated with the preceding displacive mechanisms are given elsewhere ${ }^{13}$. We were not able to find a realistic mechanism for the formation of the $\beta$ structure of plutonium from $\varepsilon-\mathrm{Pu}$.

It remains to clarify the fundamental causes which may be responsible for the complex structures found in intermediate actinides. In this respect let us note that a model of hard sphere packing of atoms does not provide a suitable geometrical description of the actinide structures, as it leads to an interpenetration, or to the absence of contact of the spheres in different crystallographic directions. For example in $\alpha-U$ a packing of spherical atoms yields a distance between the centers of the closest atoms of $2 r=3.06 \AA$ and $2 r \sqrt{ }=5.3 \AA$ respectively in the $x$ and $y$ orthorhombic directions, $r$ being the spherical atomic radius. In contrast, the gorresponding lattice parameters in these directions are respectively a $=$ $2.85 \mathrm{~A}$ and $\mathrm{b}=5.86 \mathrm{~A} . \quad$ Non-coinciding numbers are also found for most of the phases in actinide crystals when assuming a close packing of spheres. Accordingly one is entailed to suggest that non-spherical atoms should give a more coherent 


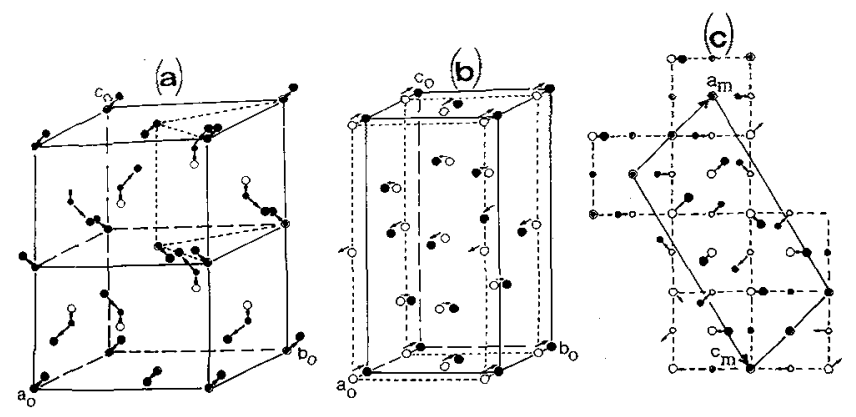

Fig.3 : (a) Formation mechanism of $\beta-$ and $\alpha-N_{p^{*}}$ Open, small and large solid circles represent the atomic positions in $\gamma^{p}, \beta$ and $\alpha-N$ respectively. Dashed lines show half of the $\gamma$-unit-cell. (b) Formation of $\gamma-\mathrm{Pu}$ from a double hcp virtual structure. Open and solid lines indicate the double hcp and $\gamma$ unit-cellls. (c) Formation of $\alpha-\mathrm{Pu}$ from $\delta-\mathrm{Pu}$ in the monoclinic b plane (010). Open and sqlid circles are the $\delta$ and $\alpha$ positions. Small and large circles differ by $\frac{b}{2}$ in the monoclinic [010] direction. Dashed and solid lines are the projections of the cubic and monoclinic unit-cells.

picture of the geometry of the structures. We will now show that an ovaloid form of the atoms is well adapted for the cristallogeometrical properties of actinides.

Fig.4.a shows the transformation mechanism from $¥-U$ to $\propto-U$ using a close packing of hard ovaloids. It consists in a rotation by $90^{\circ}$ of the ovaloids, which preserves the close packing structure. In Fig.4.b, the $\alpha$ - B transformation mechanism in $\mathrm{N}$ is represented in which the ovaloilds are turned by about 20\%. In Fig.4.c, details of the $\beta-U$ structure show that it can be understood as a packing of pseudo six-and-three fold distribution of ovaloidd atoms, resulting in an intermediate state between the $\gamma-U$ and $\alpha-U$ distributions.

An experimental confirmation of the assumed ovaloid form for uranium atoms $j_{3}$ provided by the incommensiurately modulated structure found in $\alpha-U$ below $37 \mathrm{~K}^{3,4}$, which can be interpreted as an helical distribution of the ovaloids, the pitch of the helix being incommensurate with the $\alpha-U$ translations. One of the two incommensurate wave-vector characterizing the modulated structure is close to $\mathbf{k}=\frac{1}{2} \vec{a}^{*}$ where $a^{*}$ is the orthorhombic lattice vector. Rewriting $\vec{k}$ in bcc coordinates gives $\vec{k}=\frac{1}{3}$ (OOI) which is one of the two wave-vectors proposed in our mechanism of the $\alpha-\beta$ transformation in uranium. In other words, the incommensurate structure reveals traces of the instability which contributes to the structure, i.e. it can be interpreted as a frustrated regime between the $\alpha$ and $\beta$ ovaloid distributions. 
(a)

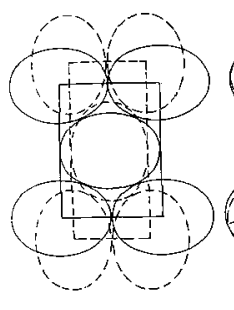

(b)

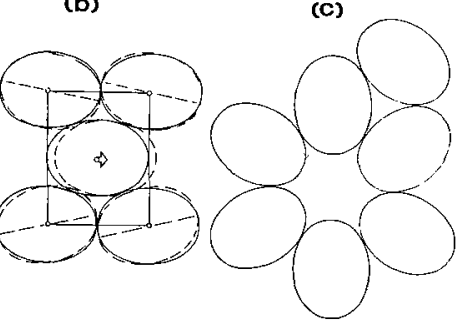

Fig. 4 : Packing of ovaloĩd atoms in uranium and neptunium. (a) $\neq-\alpha$ transformation in $U$ in projection in the bcc (110) plane. Solid and dashed ovals and lines are the projections of the $\gamma$ and $\alpha$ atoms and unit-cells respectively. (b) $\gamma-\beta$ transformations in $\mathrm{N}_{\mathrm{p}}$. Solid and dashed lines have the same meaning as in (a). The arrow symbolize the shift of the central atom of the unit-cell. (c) Characteristic motif in $-\mathrm{U}$, with an hexagonal and triangular packing of ovaloids.

\section{References}

1. Donohue, J, The structure of the elements (Wiley, New York, 1974)

2. Tonkov, E.Yu., Phase Diagrams of the Elements under Pressure (Nauka, Moscow,1979)

3. Van Smaalen, S.and George, T.F., Phys. Rev. B35 (1987), 7939

4. Marmeggi, J.C., Lander, G.H., Van Smaalen, S. Brückel, T. and Zeyen, C.M.E, Phys. Rev. B42, (1990), 9365.

5. Dmitriev, V.P., Rochal, S.B., Gufan, Yu.M. and Toledano, P., Phys.Rev.Lett 60 (1988) 1958 .

6. Dmitriev, V.P., Gufan, Yu.M., and Tolédano, P., Phys. Rev. B44 (1991), 7248

7. Stephens, D.R., Stromberg, H.D. and Lilley, D.M. J. Phys.Chem.Solids 29 (1968)815

8. Benedict, U. Handbook on the Physics of the Actinides, Ed.Freeman, A.J. and Lander, G.H. (Elsevier Sciences Publisher, Amsterdam, 1987)

9. Oelting, F.L. Rand, M.H., and Ackermann, R.J., The Chemical Thermodynamics of Actinide Elements and Compounds (IAEA, Vienna, 1976) Part. 1

10. Haire, R.G. and Baybarz, R.D., J. Phys. (France) 40, C4 (1979), 101

11. Haire, R.G. and Peterson, J.R., J. Less-Common Met. 102 (1984) 119

12. Zachariasen, W.H., Acta Cryst. 5 (1952) 660 ; and 5 (1952) 664

13. Mettout B., Dmitriev V.P., Ben Jaber M. and Toledaño P., Phys. Rev. B48 (1993)6908 